Meta

Journal des traducteurs

Translators' Journal

\title{
La responsabilité de l'interprète de conférence professionnel ou pourquoi nous ne pouvons pas écrire nos mémoires?
}

\section{Christopher Thiéry}

Volume 30, numéro 1, mars 1985

Interprétation de conférence

URI : https://id.erudit.org/iderudit/004475ar

DOI : https://doi.org/10.7202/004475ar

Aller au sommaire du numéro

Éditeur(s)

Les Presses de l'Université de Montréal

ISSN

0026-0452 (imprimé)

1492-1421 (numérique)

Découvrir la revue

Citer cet article

Thiéry, C. (1985). La responsabilité de l'interprète de conférence professionnel ou pourquoi nous ne pouvons pas écrire nos mémoires ? Meta, 30(1), 78-81.

https://doi.org/10.7202/004475ar d'utilisation que vous pouvez consulter en ligne. 


\section{LA RESPONSABILITÉ DE L'INTERPRÈTE DE CONFÉRENCE PROFESSIONNEL OU POURQUOI NOUS NE POUVONS PAS ÉCRIRE NOS MÉMOIRES}

CHRISTOPHER THIÉRY

Cela se passait pendant la visite du Président Nixon à Paris en 1969. Constantin Andronikov, que je secondais, a demandé au général de Gaulle s'il voyait un inconvénient à ce que ce soit moi qui interprète son discours à la fin du dîner à l'ambassade des États-Unis. La réaction du Président de la République m'a beaucoup frappé. Il s'est borné à demander : "Saura-t-il en répondre? ». En d'autres termes, sans mettre en doute ma compétence, il a tenu à savoir si j'étais prêt à prendre la responsabilité d'être son porte-parole en anglais.

Pour comprendre toute la signification de cette question il faut réfléchir à ce que l'on entend par interprète de conférence "professionnel ". Qu'est-ce qui le distingue d'un simple prestataire de services, occasionnel ou non ? En quoi une profession se distingue-t-elle d'un groupement basé uniquement sur des intérêts communs, comme une corporation, au sens moderne du terme? En somme, qu'est-ce qu'une profession ?

Il me semble que les trois critères suivants s'imposent - en tout cas pour les activités qui nous intéressent ici.

1. Les membres d'une profession exercent un métier dont ils vivent, au moins partiellement ; ils ont acquis, généralement moyennant une formation spécialisée, l'expertise technique requise.

2. Les membres d'une profession se conforment à une déontologie ; celle-ci régit à la fois les rapports entre eux, et avec la société. Notons tout-de-suite que cela implique l'existence d'une organisation professionnelle qui définisse les règles de la profession et en assure le respect.

3. Les membres d'une profession sont responsables de leur prestation.

Cette dernière notion est souvent perçue de façon floue; on peut cependant essayer d'en tracer les contours avec un peu plus de netteté.

Écartons d'abord toute idée de responsabilité envers autrui pour les propos que l'interprète est appelé à tenir en faisant son métier : il est évident qu'elle incombe à celui qui en est l'initiateur.

Alors responsable de quoi? et envers qui ?

En acceptant une mission l'interprète de conférence professionnel s'engage en premier lieu à transmettre de façon fidèle et complète le message dont il a la charge ; il s'agit donc d'une responsabilité envers celui dont il aura à interpréter le discours. Celui-ci se décharge sur l'interprète du soin de se faire comprendre par ceux qui n'entendent que la langue parlée par l'interprète. 
Remarquons que cette responsabilité s'exerce vis-à-vis de chacune des personnes interprétées, sans distincticn aucune. Il serait impensable de privilégier certains interlocuteurs au détriment d'autres. De même, un interprète de conférence professionnel n'est jamais, en réalité, l'interprète "de quelqu'un ". Il est inexact de parler, comme on le fait souvent, de "l'interprète de M. X, ou de Mme Y ". Même attaché à la personne, par exemple, d'un Chef d'État, l'interprète, s'il est professionnel, ne sera jamais responsable devant celui-ci uniquement. Interprétant les propos tantôt de l'un, tantôt de l'autre, il sera toujours l'interprète de la réunion, qu'il s'agisse d'un entretien, d'une conférence, d'un congrès, ou d'un tribunal. Ceci est vrai même lorsque son rôle consiste exclusivement à être le porte-parole d'une seule personne, car sa responsabilité est nécessairement double, ou du moins à deux faces : responsable envers celui dont il transmet le discours, il l'est aussi envers ceux qui l'écoutent. En acceptant sa mission il s'est engagé à leur communiquer fidèlement, complètement, les messages énoncés dans l'autre langue. Ainsi, l'interprète de conférence professionnel est, en définitive, responsable de la qualité de son travail envers l'ensemble de ce que nous appellerons, pour simplifier, la " réunion".

Deux remarques à ce propos :

- cette responsabilité s'exerce indépendamment de l'identité de l'employeur, de celui qui rémunère les services de l'interprète. A fortiori elle n'est en aucune façon affectée par la nature des liens contractuels ou le mode de rémunération convenus : salaire mensuel, salaire journalier, honoraires. "He who pays the piper does not call the tune."

- en pratique la "réunion " est le plus souvent une entité ad hoc, éphémère. Cela pose un problème, nous le verrons, pour certains aspects de la responsabilité de l'interprète qui persistent dans la durée, au-delà de l'instant même de la communication.

En assumant cette double responsabilité envers la "réunion " l'interprète de conférence professionnel accepte en fait que les participants se déchargent sur lui de leurs problèmes de communication multilingue. C'est dire qu'il lui appartient, notamment, de prendre les mesures nécessaires pour se trouver au lieu dit à l'heure voulue. Cela est évident. Ce qui l'est moins, c'est qu'il lui appartient aussi de s'assurer que les conditions matérielles (audition, visibilité, documentation, etc.) que requiert l'exécution de sa mission sont réunies. Cela vaut aussi bien pour l'interprète individuellement que pour celui qui a pour charge de constituer l'équipe. Ce n'est certes pas toujours aisé : il faut éclairer ceux qui sont mal informés, et convaincre ceux qui sont réticents, ce qui fait beaucoup de monde. L'interprète ne doit jamais perdre de vue, toutefois, qu'en agissant ainsi, loin de se présenter en demandeur, il ne fait que s'acquitter de sa responsabilité envers la "réunion". Il n'en sera que plus convaincant.

Cette position entraîne bien sûr un corollaire : si les conditions de travail ne sont pas de nature à permettre à l'interprète de s'acquitter correctement de sa mission il devra y renoncer. Cela fait partie de la "probité de la prestation" mentionnée au Code d'éthique professionnelle de l'Association internationale des interprètes de conférence (AIIC), tout comme l'obligation de refuser un engagement pour lequel on ne serait pas qualifié. En effet, comment assumer une responsabilité quelconque dans ces conditions ?

Il est un élément de la responsabilité de l'interprète de conférence professionnel qui n'échappe à personne ; le secret professionnel. Le Code de l'AIIC est particulièrement explicite à ce sujet :

Article 2

a) Les membres de l'Association sont tenus au secret professionnel total et absolu. Celui-ci doit être observé à l'égard de quiconque et concerne tout ce qui a été appris dans l'exercice de la profession à l'occasion de réunions non publiques. 
b) Ils s'interdisent de tirer un profit personnel quelconque de toute information confidentielle qu'ils auraient pu recevoir dans l'exercice de leurs fonctions d'interprète.

Tout interprète de conférence qui accepte une mission s'engage à respecter le secret professionnel, et cet engagement est pris envers non pas tel ou tel participant, mais bien envers l'ensemble de la "réunion".

Trois questions viennent imméditatement à l'esprit :

- un tribunal peut-il obliger un interprète à "trahir »le secret?

- en cas de faute d'où viendrait la sanction, et laquelle? cipants ?

l'interprète peut-il parler 20,30 ans plus tard ? Après la mort de tous les parti-

À la première question la réponse est théoriquement " oui ", pour la simple raison qu'il n'existe dans aucun pays un véritable statut de l'interprète. Sans entrer dans le débat ouvert sur la nécessité ou l'opportunité d'un tel statut, constatons qu'aucune législation n'autorise un interprète de conférence à taire certaines informations si un tribunal exige qu'il les communique. À ma connaissance toutefois la question ne s'est jamais posée, en tout cas de façon aussi explicite. Il existe même une sorte de reconnaissance de facto du secret professionnel, comme en témoigne l'intervention réussie de l'AICC dans une affaire déjà ancienne où un interprète, non membre d'ailleurs, avait travaillé (à son insu) pour une association de malfaiteurs.

La question des sanctions éventuelles nous ramène au problème déjà posé : un interprète qui aurait trahi le secret professionnel aurait de ce fait manqué à ses obligations envers l'ensemble de la "réunion ». Or, nous l'avons vu, celle-ci a souvent une existence éphémère. C'est ainsi que tout naturellement c'est l'ensemble de la profession, par le truchement de l'organisation professionnelle, qui se substitue à ceux qui seraient lésés. Il y a transfert ou plutôt dédoublement, de l'obligation souscrite par l'interprète : celuici devient responsable de ses actes également devant ses pairs. Cette solidarité s'ajoute à l'obligation de confraternité professionnelle au sens large du terme.

Quant à la nature des sanctions, il n'y a pas, fort heureusement, de jurisprudence. Il est très probable que la peine serait la plus forte prévue : l'exclusion, non seulement de l'organisation professionnelle, mais en pratique de la profession. Un interprète de conférence qui trahirait le secret professionnel ne perdrait pas simplement un employeur, il perdrait son gagne-pain.

De ces considérations découle la réponse à la troisième question : Même après la mort des interlocuteurs l'interprète de conférence professionnel n'est pas pour autant délié de ses obligations en matière de secret professionnel, ne serait-ce que parce que celles-ci ont également été contractées envers ses pairs. L'organisation professionnelle peut-elle lever, 30 ans après, ces obligations ? Elle serait bien mal avisée de le faire, car la divulgation en 1984 de faits remontant à 1954 ne ferait peut-être pas grand mal aux acteurs de l'époque, surtout s'ils sont morts, mais elle ne manquerait pas de rejaillir sur l'image de la profession et de porter atteinte au climat de confiance absolue qui est une condition majeure de son exercice. Ce sont les interprètes exerçant en 1984 qui en pâtiraient.

C'est ainsi qu'il est exclu, me semble-t-il, qu'un interprète de conférence professionnel écrive ses mémoires.

Et pourtant il existe des "mémoires d'interprètes » : ceux de Schmidt, Dolmann Birse, Walters, pour ne citer que les plus connus. Certains de ces ouvrages sont passionnants - et fort indiscrets. Leur analyse déborderait le cadre du présent article, mais une conclusion s'impose d'emblée : il s'agit chaque fois de personnes qui ont "fait fonction " d'interprète, souvent de façon fortuite dans un premier temps. Ils l'ont fait, semble-t-il, avec efficacité, à en juger par les remarques pertinentes sur le métier dont leurs livres 
abondent. Mais à aucun moment ils ne se considèrent membres d'une profession. On chercherait en vain des expressions de solidarité avec l'ensemble de ceux qui font le même travail. Dans certains cas il faut dire que la profession n'avait pas encore pris forme. Dans d'autres l'auteur se considérait bel et bien comme membre d'une autre profession - pour Vernon Walters, par exemple, celle du renseignement, sur laquelle il est d'ailleurs fort discret. En tout cas avec le passage du temps, la disparition des interlocuteurs, et peut-être en fonction de considérations politiques, ces auteurs se sont sentis libres de parler. C'est que le transfert ne s'est pas fait : à aucun moment ils ne se sont sentis responsables devant leurs pairs, devant tous ceux qui exercent la même activité. C'est toute la différence entre le prestataire de services et le membre d'une profession.

L'interprétation de conférence est une profession récente. Elle a pourtant une réalité, une cohésion, une déontologie que nombre de professions plus anciennes pourraient lui envier. Encore faut-il que les membres de la profession aient clairement conscience des responsabilités très précises qu'ils assument en acceptant une mission : c'est ainsi qu'ils joueront pleinement leur rôle, humble mais vital, dans la société humaine, et qu'ils connaîtront pour eux-mêmes les satisfactions intellectuelles et morales qui échappent à ceux qui se bornent à "faire l'interprète ". 